\title{
Effect of systematic and random flow measurement errors on history matching: a case study on oil and wet gas reservoirs
}

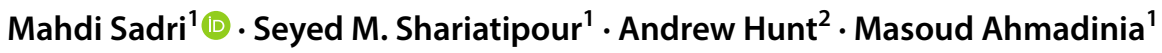

Received: 20 November 2018 / Accepted: 9 April 2019 / Published online: 19 April 2019

(c) The Author(s) 2019

\begin{abstract}
History matching is the process of modifying a numerical model (representing a reservoir) in the light of observed production data. In the oil and gas industry, production data are employed during a history matching exercise in order to reduce the uncertainty in associated reservoir models. However, production data, normally measured using commercial flowmeters that may or may not be accurate depending on factors such as maintenance schedules, or estimated using mathematical equations, inevitably has inherent errors. In other words, the data which are used to reduce the uncertainty of the model may have considerable uncertainty in itself. This problem is exacerbated for gas condensate and wet gas reservoirs as there are even greater errors associated with measuring small fractions of liquid. The influence of this uncertainty in the production data on history matching has not been addressed in the literature so far. In this paper, the effect of systematic and random flow measurement errors on history matching is investigated. Initially, 14 production data sets with different ranges of systematic and random errors, from 0 to $10 \%$, have been employed in a history matching exercise for an oil reservoir and the results have later been evaluated based on a reference model. Subsequently, 23 data sets with errors ranging from 0 to $20 \%$ have been employed in the same process for a wet gas reservoir. The results show that for both cases systematic errors considerably affect history matching, while the effect of random errors on the considered scenarios is seen to be insignificant. Although reservoir model parameters in the wet gas reservoir were not as sensitive to the flow measurement errors as in the oil reservoir, for both cases, the future production forecast was significantly affected by the errors. Permeability was seen to be the most sensitive history matching parameter to the flow measurement errors in the oil reservoir, while for the wet gas reservoir, the most sensitive parameter was the forecast of future oil and gas production. Finally, considering the noticeable effect of systematic errors on both cases, it is suggested that flowmeter calibration and regular maintenance is prioritised, although the subsequent economic cost needs to be considered.
\end{abstract}

Keywords Flow measurement $\cdot$ History matching $\cdot$ Systematic error $\cdot$ Random error $\cdot$ Wet gas reservoir

\section{Introduction}

The knowledge of reservoir management has dramatically improved. Managing hydrocarbon reservoirs to maximise the profit from them, which had a limited knowledge and involved just simple calculations in the early years of the oil and gas industry, has become a complicated dynamic process of setting goals, decision making, implementing,

Mahdi Sadri

Sadrim@uni.coventry.ac.uk

1 Fluid and Complex Systems Research Centre, Coventry University, Coventry, UK

2 Atout Process Limited, Southampton, UK monitoring, analysing data, and modifying decisions (Satter et al. 1994). Reservoir management in its present form needs a multidisciplinary approach and the integrated application of different technologies and professional software. In this process, a large amount of data are recorded and analysed and engineers need to deal with numerous uncertainties. Trice Jr and Dawe (1992), Satter et al. (1994), Al-Hussainy and Humphreys (1996), and Thakur (1996) have explained principles of reservoir management in their publications. Recently, the concept of closed-loop reservoir management (CLRM) has been introduced in the literature and a number of studies have been published on this title (Barros et al. 2016; Hanssen et al. 2017; Jansen et al. 2005, 2009; Lorentzen et al. 2009; Wang et al. 2009). CLRM (Fig. 1) is a combination of history matching and model-based optimisation, and its aim is 


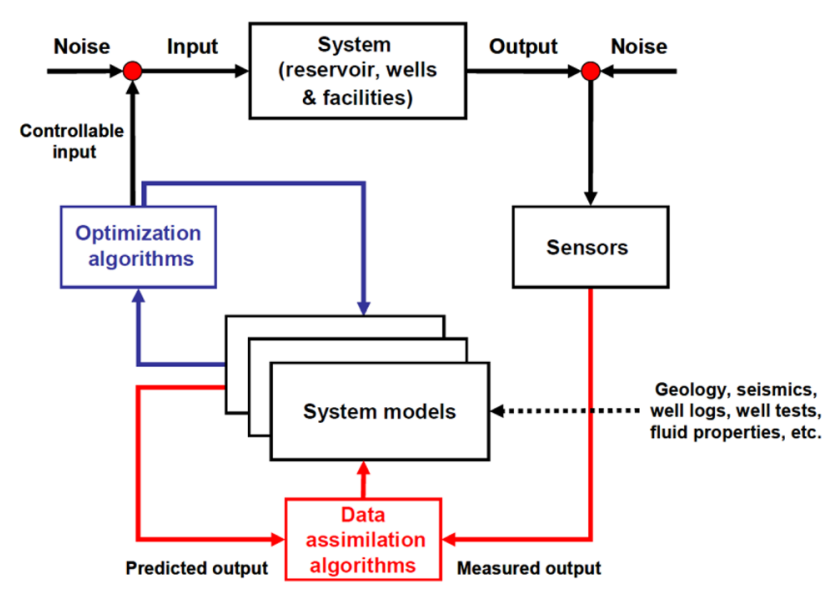

Fig. 1 Closed-loop reservoir management (Jansen et al. 2009). The uncertainty in the reservoir output data affects history matching (data assimilation) and reservoir management. A part of this uncertainty is the errors in the recorded production data

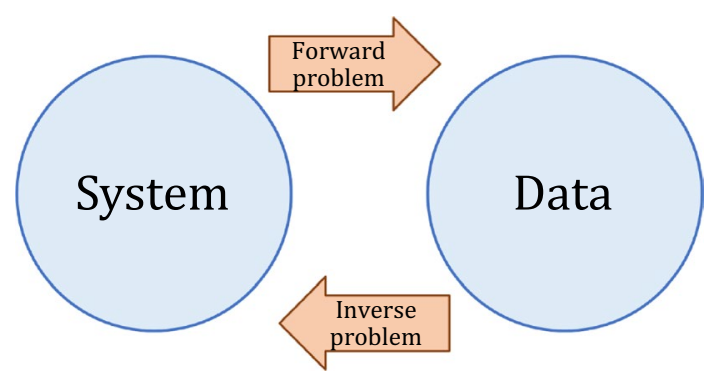

Fig. 2 Forward and inverse problems. In an inverse problem, such as history matching, the characteristics of an unknown system are estimated based on its observed output data

to change reservoir management from a periodic to a nearcontinuous process (Jansen et al. 2009). As shown in Fig. 1, history matching plays an important role in the management process as it has a direct effect on the reservoir model and an indirect effect on the decisions and plans for the reservoir through its impact on the model and on the reservoir optimisation. Therefore, perhaps we can name history matching as "the heart of CLRM" since it synchronises the two reservoirs (actual reservoir and reservoir model) in the loop.

History matching is an inverse problem. In a forward problem, the output of a system is calculated based on the characteristics of the system (Fig. 2). In an inverse problem, the system is unknown and the observed output of the system is used to determine its characteristics (Kirsch 2011). However, the output data usually have inherent errors that affect the calculations. As a consequence, the obtaining system characteristics might be different from the actual ones. In history matching, the system is a reservoir and the output is its production data. The difference is that the reservoir is not completely unknown and an initial model is available based on the information obtained from other sources such as seismic data, well testing, and laboratory experiments on the characteristics of the reservoir rock and fluid samples. However, since this initial model is highly uncertain, the production data are used to mitigate the model uncertainties. History matching is widely used in the oil and gas industry, and many different methods of performing it have been published in the literature (Chakra and Saraf 2016; Hamdi et al. 2015; Makhlouf et al. 1993; Obidegwu et al. 2017; Oliver and Chen 2011; Oliver et al. 2008; Tunnish et al. 2018).

As stated above, production data are employed in history matching to reduce the uncertainty of the reservoir model. However, production data (oil and gas production rates, water cuts, and downhole or wellhead pressures) similarly have inherent uncertainty. Any observed data inevitably contain errors, and the extent of an error depends on the estimation method or the measurement equipment which is employed to gather the data. Therefore, history matching does not merely deal with the uncertainty in the system, but also with the uncertainty in its own inputs which can potentially affect the results. Although different types of reservoir uncertainty have been comprehensively studied previously (Abdollahian et al. 2018; Babak and Deutsch 2008; Habib et al. 2017; Mozaffari et al. 2017; Stephen and Macbeth 2008; Tavassoli et al. 2004; Xu et al. 2018), the uncertainty in the observed data has not drawn the attention of researchers so far.

Oil, gas, and water production flow rates are the main observed data sets used in history matching. In different oil and gas fields, different methods are used to record production flow rates. In many cases, production from different wells is commingled and the total production is sent to the separation unit. The single phase flowing out of the separation unit is measured by flowmeters subsequently. Having the total flow rate of all wells, engineers then allocate flow rates to each well based on allocation factors. Allocation factors are normally determined based on flow rate measurements from intermittent tests on individual wells. To perform the tests, operators disconnect individual wells from the main production pipe and send the flow rate of the well to a test separator. As a result, they can measure production flow rates for individual wells and hence calculate the proportion of the production of each well to the total production. The gap between two tests varies for different fields, but normally, the test is not undertaken more than once a month. The uncertainty in allocation methods is large since the actual proportion of the production of each well to the total production does not remain the same as the measured one. Moreover, there is no guarantee that the production conditions over the test time are the same as the conditions during the normal production. The principles of different methods of allocation have been explained by the Energy Institute (2012). The focus of many publications on allocation is its application in hydrocarbon accounting (Cramer et al. 2011; Kaiser 
2014; Pobitzer et al. 2016). There is a dearth of publications on the application of allocation data in reservoir analysis, reservoir management and history matching. Among the latter publications is the work of Bergren et al. (1997). They reported their successful experience in employing an allocation system including computers, communications hardware, and software for both hydrocarbon accounting and reservoir management for Prudhoe Bay oil field in Alaska. In another research study, Marshall et al. (2018) investigated the effect of random errors in production data on forecasted hydrocarbon recovery. They showed that in a case where the reservoir model is selected incorrectly due to the errors, it can have a significant effect on the estimated recovery factor and reservoir parameters which are obtained in well testing.

Another method that is currently employed in some fields, especially for offshore ones, is the use of multiphase flowmeters (MPFM) for individual wells. In this method, although the production data of individual wells are recorded with a higher accuracy, the data still have some errors. The error is larger for gas reservoirs where gas void fractions are greater than $90 \%$ (Leeson et al. 2001). MPFMs normally struggle to recognise small fractions of liquid. Therefore, for a gas void fraction greater than $90 \%$, the inaccuracies rapidly rise with the increasing percentage of gas. Generally, flow measurement in wet gas and gas condensate reservoirs is more challenging than oil reservoirs and the flow measurement data for gas reservoirs normally include more uncertainty (Letton and Hall 2012). Falcone et al. (2002) undertaken a thorough research on the applications of MPFMs in the oil and gas industry. A book on principles and applications of MPFMs has been published based on their research later (Falcone et al. 2009).

Flowmeters exhibit two types of error: systematic and random. Random errors shift each measurement by a random amount up to the error specification of the flowmeter in a random direction. Therefore, different measured values are obtained when a measurement is repeated several times for a constant quantity. Random errors have no pattern, and they are unpredictable. Although there is no way to have zero random error and the existence of random errors in the measured data is unavoidable, it is possible to increase the precision of the flowmeter and reduce the error specification by using new flowmetering technologies (Tombs et al. 2006). Random errors tend to be normally distributed. They can be analysed statistically and explained in terms of their mean (Eq. 1) and standard deviation (Eq. 2).

$$
\begin{aligned}
& \bar{x}=\frac{1}{n}\left(\sum_{i=1}^{n} x_{i}\right) \\
& \mathrm{SD}=\sqrt{\frac{\sum_{i=1}^{n}\left(x_{i}-\bar{x}\right)^{2}}{n-1}}
\end{aligned}
$$

where $\bar{x}$ refers to mean, SD denotes standard deviation, $x_{i}$ refers to the error for each data point and $n$ denotes the total number of data points.

In this article, the word "precision" has been used to describe the magnitude of the random errors qualitatively. In technical terms, a more precise flowmeter has smaller random errors. On the other hand, systematic errors are normally predictable and they usually exhibit a pattern. Systematic errors shift all of the measurements in the same direction and by the same magnitude. The error is typically constant or proportional to the true value of the measured quantity. Since the shift of the data is in one direction, systematic errors do not have a zero mean. Another difference between systematic and random errors is that systematic errors can be avoided by identifying and eliminating their causes, these primarily being improper calibration and poor maintenance. Flowmeters are normally subjected to high pressure and temperature, friction with fluids, and deposition of asphaltene, wax, and scale. These factors and other similar environmental impacts change the operational conditions of a flowmeter (Lindsay et al. 2017; Liu et al. 2017). Therefore, they need to be calibrated and maintained regularly to be able to work within the desired error specifications and it takes a part of annual operational costs of oil and gas companies. The term that has been used in this article to explain the extent of systematic error is "trueness". "Accuracy" is another more general technical term that includes both types of errors. An accurate flowmeter is a flowmeter with a high precision and trueness (i.e. low systematic and random error). The terminology is the same as most technical articles on errors. Figure 3a shows the different possible states of a data set in terms of its trueness and precision. More information about systematic and random errors has been presented by Taylor (1997). Figure 3b shows the data sets which were employed for the oil cases in this research. The comparison between Fig. 3a, b suggests that each data set based on its random and systematic error represents a different level of precision and trueness. More details about Fig. $3 b$ and the generation of the data sets have been presented below in the oil reservoir section of this article.

So far, the focus of many oil and gas industry researchers and professionals has been on investigating the effects of flow measurement errors on custody transfer and fiscal measurement (i.e. hydrocarbon accounting). Custody transfer is when oil or gas is transferred from one operator to another. Fiscal measurement is a more general term. It includes any flow measurement used to determine the financial value of the delivered oil and gas. In these cases, the accuracy of the measurements is indeed important. Therefore, countries have precise regulations and standards for custody transfer and fiscal measurement that determines the acceptable error ranges. As a result, operators use approved flowmeter technologies to meet these 
Fig. 3 a Different states of a data set in terms of its trueness and precision and; $\mathbf{b}$ the error values of the data sets employed in this research (each blue point represents both systematic and random error values for one of the data sets). The defined data sets in $\mathbf{b}$ represent different states in $\mathbf{a}$
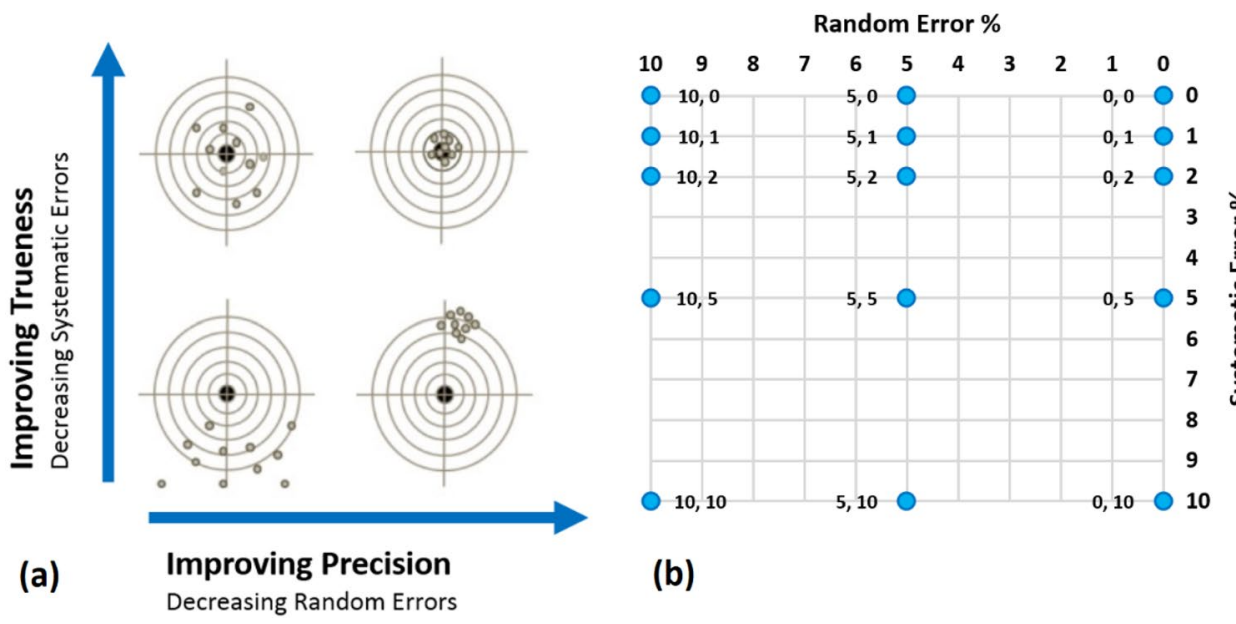

(b) regulations. Several publications about flow measurement regulations for custody transfer and fiscal measurement are available online. For instance, Guidance Notes for Petroleum Measurement (2015) explains the regulations in the UK. So far, the role of flow measurement in reservoir management has not drawn the attention of professionals as much as its role in fiscal measurements. However, the new methods of reservoir management are strongly dependent on data analysis and as a result they are highly sensitive to the quality of the data. As shown in Fig. 1, the output data from the actual reservoir are inevitably noisy and this noise and any other error in the observed data impacts on the whole process of reservoir management, including history matching and reservoir optimisation. The quality of the data also indirectly affects the hydrocarbon recovery by affecting the reservoir management and decision-making process. Therefore, it is important to have an idea of the required quality for the data which can guarantee a good management over the reserves and maximised oil and gas recovery. In this research, the effect of systematic and random flow measurement errors on history matching has been investigated. Production data are a main group of data which is employed in reservoir management. Investigating the effects of flow measurement errors in production data on history matching opens up new ways to undertake further research on the effects of flow measurement errors on reservoir management and hydrocarbon recovery. In a previous study, we showed that flowmeters which have errors just in one direction (i.e. positive or negative) cause more errors in the results of history matching compared to when they have errors in both directions (Sadri et al. 2017). In this paper, as a more general investigation, the effect of systematic and random flow measurement errors on history matching in an oil and a wet gas reservoir is addressed. In the following lines, first, the methodology of this work including the details of the simulation models, the prepared Matlab ${ }^{\circledR}$ code, and the error data sets has been explained, the results presented and discussed, and finally the conclusions and suggestions have been briefly stated.

\section{Methodology}

Two case studies on two synthetic reservoirs, an oil and a wet gat reservoir, have been undertaken in this research to investigate the effects of systematic and random flow measurement errors on history matching.

\section{Oil reservoir}

For the oil reservoir case, a reference reservoir model with the characteristics shown in Table 1 was employed in the Schlumberger ECLIPSE simulator to produce reference production data (oil, gas, and water production rates) over 10 years.

The data were then imported into the Matlab ${ }^{\circledR}$ software and a Matlab ${ }^{\circledR}$ code generated 15 data sets with different ranges of systematic and random error, as shown in Fig. $3 b$. Ten per cent was chosen as the highest error since most of the publications in the literature report flow measurement

Table 1 Characteristics of the reference oil reservoir

\begin{tabular}{ll}
\hline Initial reservoir pressure & $304.20 \mathrm{Bar}$ \\
Porosity & 0.18 \\
Horizontal permeability & $60 \mathrm{mD}$ \\
Vertical permeability & $10 \mathrm{mD}$ \\
Saturation pressure of reservoir hydrocarbon & $386.11 \mathrm{Bar}$ \\
Density of oil at the surface conditions & $721 \mathrm{~kg} / \mathrm{m}^{3}$ \\
Density of water at the surface conditions & $1009 \mathrm{~kg} / \mathrm{m}^{3}$ \\
Density of gas at the surface conditions & $1.12 \mathrm{~kg} / \mathrm{m}^{3}$ \\
\hline
\end{tabular}

These characteristics were employed to build the reservoir model 
errors for oil wells which are within this range. These data sets were later used as observed production data in history matching. Random errors have been produced using randomly generated numbers within the specified ranges (i.e. $0 \%, 5 \%$, and 10\%) in both the positive and negative directions. For instance, when the error specification was 5\%, random errors could take any value between $-5 \%$ and $+5 \%$. However, systematic errors were set to fixed percentages (i.e. $0 \%, 1 \%, 2 \%, 5 \%$, and $10 \%$ ) and their values were proportional to the reference values. After creating the data sets, the Matlab ${ }^{\circledR}$ code generated RSM files (a format which can be imported into Eclipse for further simulations and history matching) including the observed data. The code can also perform a statistical analysis and report the results in terms of the mean (Eq. 1) and standard deviation (Eq. 2) of the data sets in an Excel file. The statistical information about the data sets used in this work is shown in Table 2.

In the next stage of the work, the observed data (i.e. reference data with errors) were used in history matching to modify an uncertain reservoir model. The only differences between the uncertain model and the reference model were the values for porosity and permeability. These values were 0.28 and $40 \mathrm{mD}$ for the uncertain model, respectively. Finally, the data from the modified model were compared to the data from the reference model to show the effect of the systematic and random flow measurement errors on the results of the history matching (i.e. estimated porosity, permeability, oil and gas production). This process was undertaken for all the data sets with different random and systematic errors, and the results were compared and analysed. The systematic and random errors were defined so that they represent different states of a flowmeter based on its trueness and precision, as shown in Fig. 3. The density of the data sets around $(0,0)$ point was higher because in high precision and high trueness even a $1 \%$ change in the error might have a significant effect. Also, the number of the chosen values for systematic error was greater than random errors because initially we expected to see a more significant effect due to systematic errors; an expectation which was later proved to be correct based on the results.

In this research, the traditional method of history matching has been used to modify the uncertain reservoir model. In the traditional method, the best match between the simulation results and the observed data is used for reservoir model modifications. The best match is obtained by performing the simulations and comparing the simulation results to the observed data in an iterative procedure. In each iteration, the sum of the squared residuals (difference between the observed and simulated values) is calculated and compared to that of the previous iteration. The aim of this method (least-squares method) is to minimise the mentioned calculated value. When the difference between two calculated values in two consequent iterations is less than a specified
Table 2 The details of the errors in different data sets which were used in the history matching

\begin{tabular}{|c|c|c|c|c|}
\hline $\begin{array}{l}\text { Data set } \\
\text { number }\end{array}$ & $\begin{array}{l}\text { Systematic } \\
\text { error }(\%)\end{array}$ & $\begin{array}{l}\text { Random } \\
\text { error (\%) }\end{array}$ & $\begin{array}{l}\text { Mean of } \\
\text { errors (\%) }\end{array}$ & $\begin{array}{l}\text { Standard devia- } \\
\text { tion of errors (\%) }\end{array}$ \\
\hline 1 & 1 & 0 & 1.00 & 0.00 \\
\hline 2 & 2 & 0 & 2.00 & 0.00 \\
\hline 3 & 5 & 0 & 5.00 & 0.00 \\
\hline 4 & 10 & 0 & 10.00 & 0.00 \\
\hline 5 & 20 & 0 & 20.00 & 0.00 \\
\hline 6 & 0 & 5 & -0.07 & 2.92 \\
\hline 7 & 1 & 5 & 0.93 & 2.92 \\
\hline 8 & 2 & 5 & 1.93 & 2.92 \\
\hline 9 & 5 & 5 & 4.93 & 2.92 \\
\hline 10 & 10 & 5 & 9.93 & 2.92 \\
\hline 11 & 20 & 5 & 19.93 & 2.92 \\
\hline 12 & 0 & 10 & -0.13 & 5.84 \\
\hline 13 & 1 & 10 & 0.87 & 5.84 \\
\hline 14 & 2 & 10 & 1.87 & 5.84 \\
\hline 15 & 5 & 10 & 4.87 & 5.84 \\
\hline 16 & 10 & 10 & 9.87 & 5.84 \\
\hline 17 & 20 & 10 & 19.87 & 5.84 \\
\hline 18 & 0 & 20 & -0.27 & 11.68 \\
\hline 19 & 1 & 20 & 0.73 & 11.68 \\
\hline 20 & 2 & 20 & 1.73 & 11.68 \\
\hline 21 & 5 & 20 & 4.73 & 11.68 \\
\hline 22 & 10 & 20 & 9.73 & 11.68 \\
\hline 23 & 20 & 20 & 19.73 & 11.68 \\
\hline
\end{tabular}

The data sets in the lighter shaded rows have been used for both oil and wet gas reservoirs and the data sets in the darker shaded rows have been used just for the wet gas reservoir. These data sets in addition to the reference production data have been employed to generate the observed data in the history matching

value (e.g. 0.1), the iterative procedure is stopped and the match is considered as the best possible one. Since the parameters of the reservoir model (in our case the porosity and permeability) are updated in each iteration to gain a better match, in fact, the model modification is also performed in the iterative procedure at the same time. Therefore, the model of the last iteration is considered the most up to date reservoir model and used for production forecast. We refer to this model as the "modified model" in this article.

Porosity and permeability were chosen as the parameters to be modified because they are among the most important characteristics of a reservoir that can affect its production forecast. The other characteristics of the uncertain model were kept the same as the reference model to make the problem simple and enable us to see the pure effect of flow measurement data on the modifications and future forecast In the final step, the porosity and permeability of the modified model and the oil and gas production forecast for the next 20 years have been compared to those of the reference model

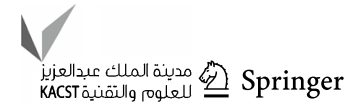


and the error of these parameters has been reported to show the effect of flow measurement errors on history matching. The errors in porosity, permeability, and forecasted oil and gas production were calculated based on Eq. 3 .

$E_{\mathrm{P}}=\left|\frac{v_{\mathrm{est}}-v_{\text {ref }}}{v_{\text {ref }}} \times 100\right|$

where $E_{\mathrm{P}}$ is the parameter error $(\%), v_{\text {est }}$ is the estimated parameter value, and $v_{\text {ref }}$ is the reference parameter value.

Figure $4 a-c$ shows the obtained matches between the simulation results (oil, gas and water production rates) and the data set with $5 \%$ systematic error and $10 \%$ random error after the history matching. The results of the reference oil reservoir have also been illustrated for a comparison. The
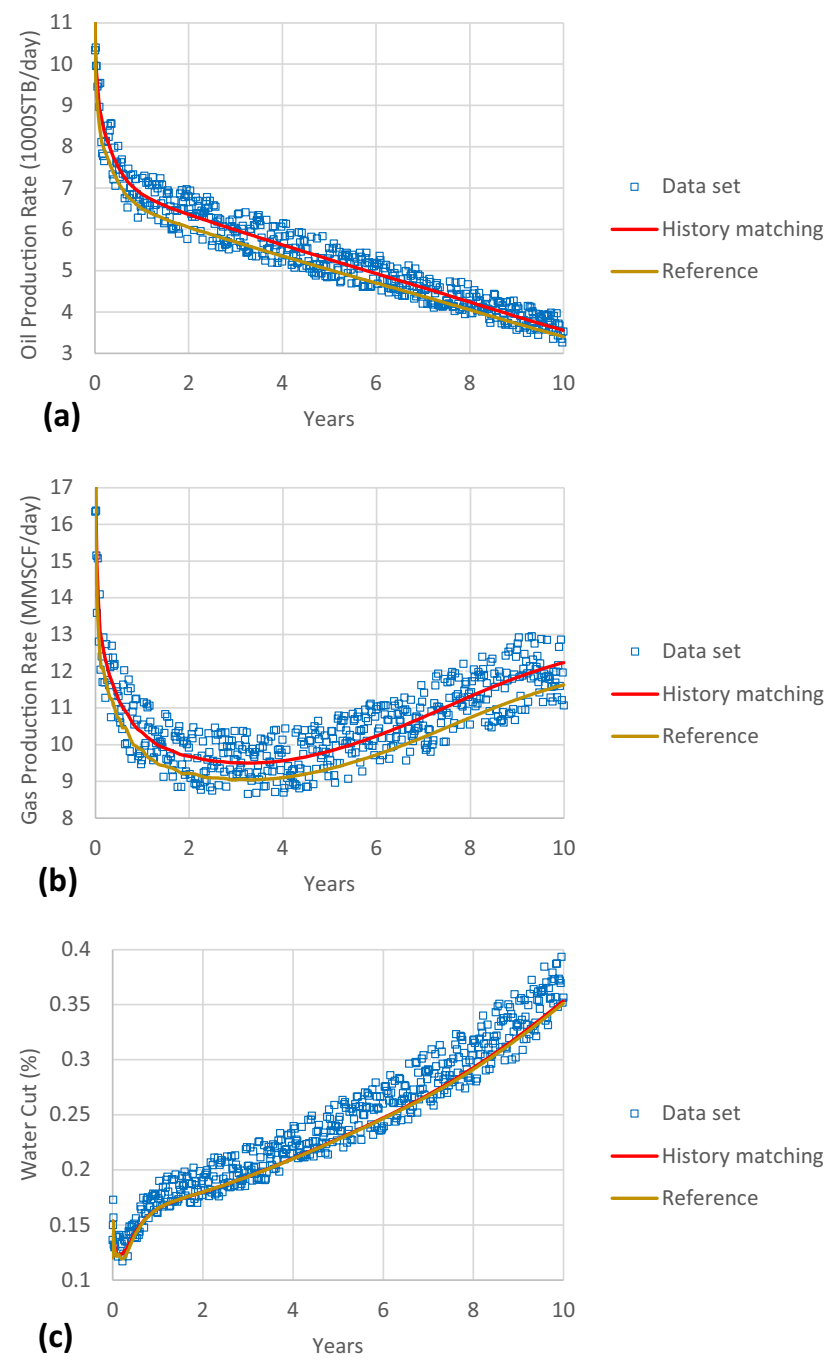

Fig. 4 The history matching results of the oil reservoir for: $\mathbf{a}$ oil; $\mathbf{b}$ gas, and; $\mathbf{c}$ water production rates based on the data set with $5 \%$ systematic and $10 \%$ random error. The figures also show the reference production rates. The gap between the two lines shows the effect of the errors on history matching gap between the results of the history matching and the reference reservoir clearly show that the errors have affected the history matching.

\section{Wet gas reservoir}

The same procedure for the oil reservoir was undertaken for a synthetic wet gas reservoir, with characteristics shown in Table 3. As stated above, measuring flow rates in wet gas reservoirs is more challenging than oil reservoirs due to the small fraction of producing liquid compared to gas. Therefore, there is normally more uncertainty associated with observed data for wet gas reservoirs than oil reservoirs (Letton and Hall 2012). To address this issue in the research, the range of systematic and random flow measurement errors in the observed data for the wet gas reservoir was increased to $20 \%$. As a result, 23 observed data sets with different values of systematic and random flow measurement errors were employed in the history matching for the wet gas reservoir. The statistical information of the data sets is shown in Table 2.

\section{Results and discussion}

Figure 5a-d shows the final errors in the oil reservoir history matching results from different observed data sets. The figures illustrate the errors in the simulated oil production, gas production, porosity and permeability, respectively.

The plots clearly illustrate the substantial effect of systematic errors on history matching, with a contradictory suggestion that the effect of random errors is insignificant. While history matching errors of all of the considered parameters (oil production, gas production, porosity, and permeability) are less than $2 \%$ when systematic error is $0 \%$ and random error is $10 \%$, they increase to more than $9 \%$ for the opposite case when systematic error is $10 \%$ and random error is $0 \%$. Therefore, based on the results, an increase in the systematic error (i.e. decreasing trueness) is seen to cause a significant increase in the history matching error for all the investigated parameters. However, all the simulation results show that

Table 3 Characteristics of the reference wet gas reservoir

\begin{tabular}{ll}
\hline Initial reservoir pressure & $302.06 \mathrm{Bar}$ \\
Porosity & 0.18 \\
Horizontal permeability & $60 \mathrm{mD}$ \\
Vertical permeability & $10 \mathrm{mD}$ \\
Density of liquid hydrocarbon at the surface conditions & $640 \mathrm{~kg} / \mathrm{m}^{3}$ \\
Density of water at the surface conditions & $1009 \mathrm{~kg} / \mathrm{m}^{3}$ \\
Density of gas at the surface conditions & $0.84 \mathrm{~kg} / \mathrm{m}^{3}$
\end{tabular}

These characteristics have been employed to build the reservoir model 
Fig. 5 Final errors of the history matching in estimating: a oil production; $\mathbf{b}$ gas production; c porosity; and d permeability for all the employed data sets for the oil reservoir ("sys" and "rand" refer to systematic and random errors, respectively). Systematic error has had a considerable effect on the history matching (a)

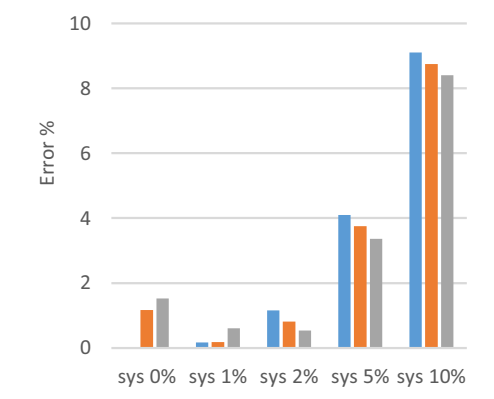

(c)

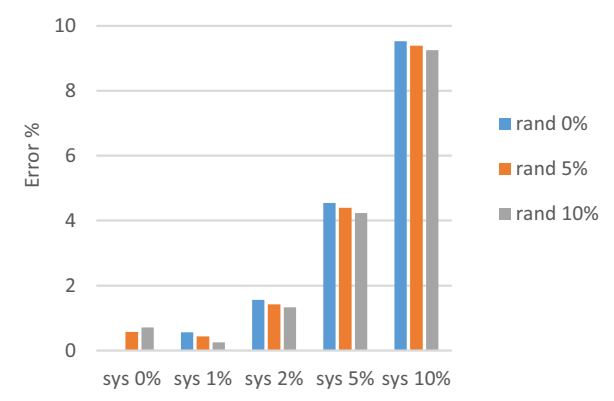

(b)

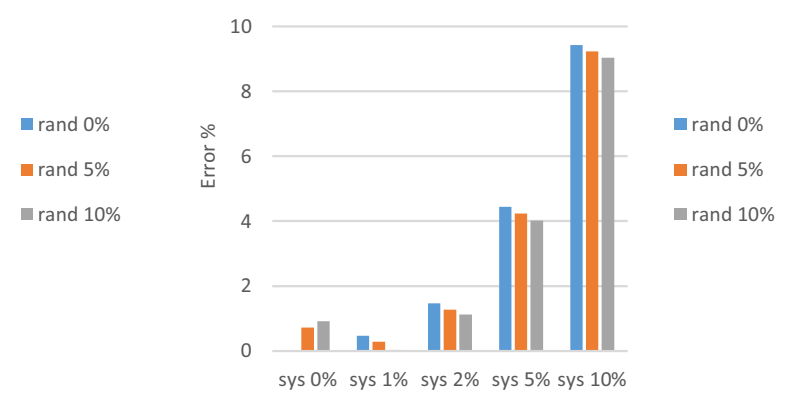

(d)

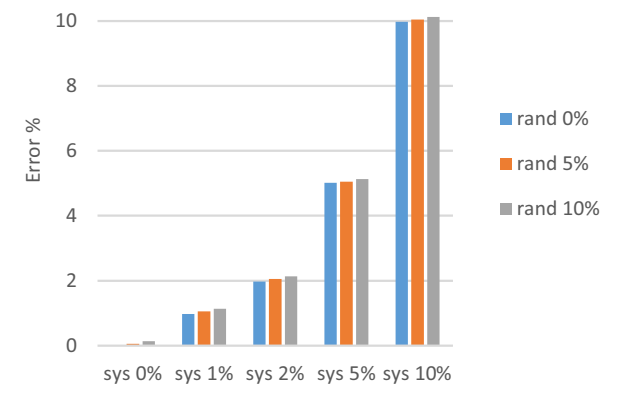

increasing random error (i.e. decreasing precision) does not have a major effect on history matching. Unexpectedly, for the oil production, gas production, and porosity, we see a decrease in the history matching errors with the increase in the random error when the systematic error is more than $2 \%$. Therefore, the results show that in this region, not only does the lower precision of the flowmeter not increase the error in history matching, but the results are seen to be improved by a dampening in the effect of the systematic errors. This dampening effect can be a consequence of the normal distribution of the random errors.

The results of three out of four parameters (oil production, gas production, and porosity) show a decreasing trend in the history matching error when the systematic error is small (i.e. from 0 to $1 \%$ ). The trend then increases for larger systematic errors (i.e. from 2 to $10 \%$ ). Therefore, it can be concluded that for these three parameters the effect of random error is dominant when systematic error is small (i.e. less than $2 \%$ in this case). However, beyond $2 \%$, the systematic error has a dominant effect. The plot for the fourth parameter (permeability), though, shows a continuous increase in history matching error for all the range of the systematic error (i.e. from 0 to 10\%). In contrast to the other three parameters, increasing the random error also leads to a continuous increase in the permeability error for the whole range. All these results, in addition to the higher value of permeability errors compared to the errors of the other parameters, suggest that the estimated permeability is more sensitive to flow measurement errors. Therefore, for the permeability even the effect of a $1 \%$ increase in the systematic error can clearly be seen in the plot.

Figure 6a-d shows the final errors in the history matching results for the gas reservoir from different observed data sets. Similar to the oil reservoir, the plots show errors in hydrocarbon liquid production, gas production, porosity, and permeability.

In wet gas reservoirs, the hydrocarbon under the reservoir conditions (reservoir pressure and temperature) is in the gas phase. The liquid hydrocarbon appears in their production as a result of the low pressure on the surface. Since no liquid hydrocarbon is formed and accumulated in the formation around the wells (inside wet gas reservoirs) during production, the composition of the producing hydrocarbon does not change over time. Therefore, in contrast to oil or gas condensate reservoirs, the gas to liquid ratio (GLR) remains the same during the life of a wet gas reservoir. As a result of the constant GLR in wet gas reservoirs, the error in hydrocarbon liquid and gas production forecast is exactly the same because their ratio remains the same. This is the reason why Fig. 6a, $b$ shows similar errors for the liquid and gas production forecasts. More information about characteristics of wet gas reservoirs is presented by Ahmed (1989), McCain (1990) and Dandekar (2013).

The results of the history matching exercise for the wet gas reservoir generally agree with the results obtained from the oil reservoir. Similar to the previous case, the effects of the random flow measurement errors were seen to be insignificant but the effect of the systematic errors are seen to be

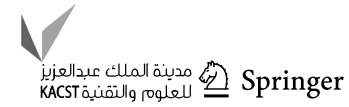


Fig. 6 Final errors of the history matching in estimating: a hydrocarbon liquid production; b gas production; c porosity; and $\mathbf{d}$ permeability for all the employed data sets for the wet gas reservoir ("sys" and "rand" refer to systematic and random errors, respectively). Systematic error has had a considerable effect on the history matching
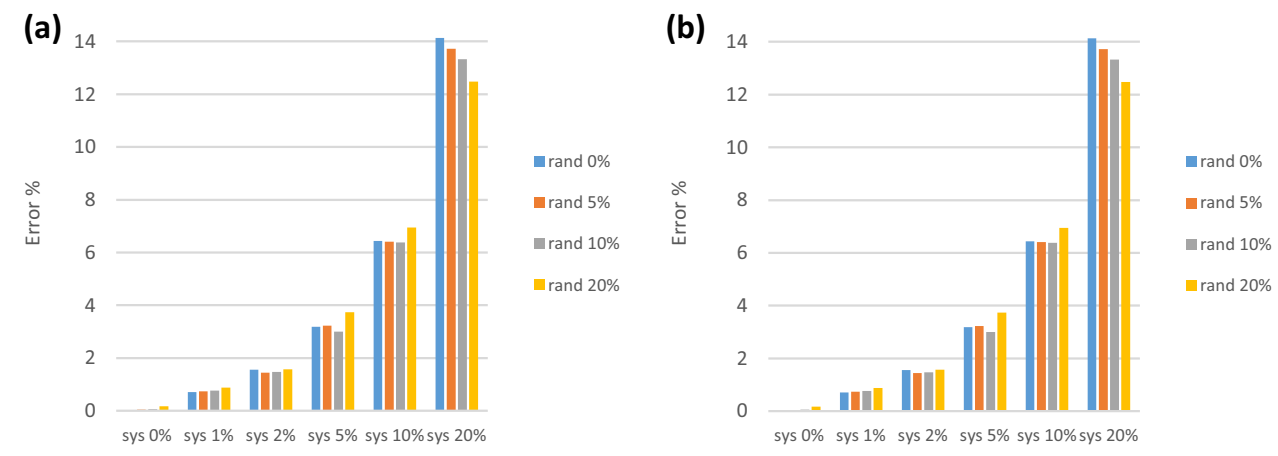

(c)

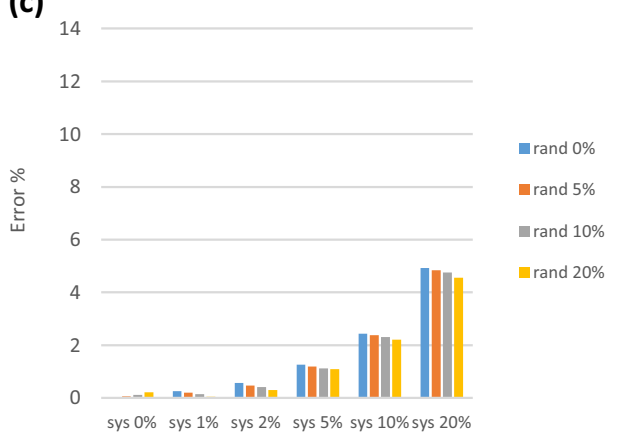

(d)

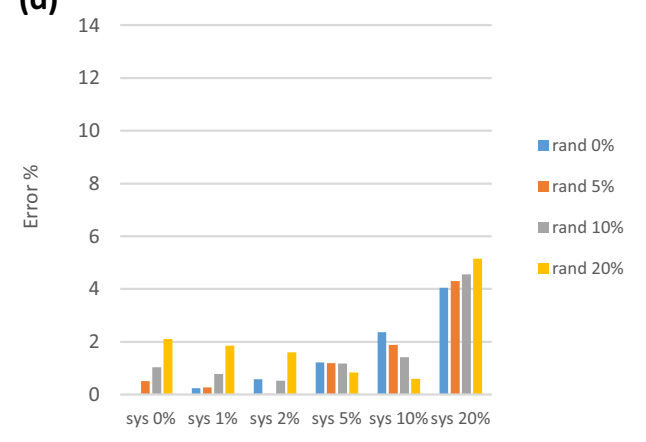

considerable. In considering all the plots in Figs. 5 and 6, it is observed that there is no general trend in the changes of the history matching error as a function of the random error. It occurs due to the nature of random errors since they unsystematically deviate the data points towards both directions. Therefore, the total effect of the random errors may boost the effect of the systematic error if they are in the same direction or balance it if they are in opposite directions. Although greater errors (up to 20\%) were applied to the wet gas data sets, the effect of the flow measurement errors on the wet gas reservoir model parameters (i.e. porosity and permeability) was seen to be less than their effect on the oil reservoir model. For instance, the largest error in the oil reservoir model parameters was $10.13 \%$ which occurred for an estimated permeability based on the data set with $10 \%$ systematic and $10 \%$ random error. This value in the wet gas reservoir results is just $5.15 \%$ for estimated permeability based on the data set with $20 \%$ systematic and $20 \%$ random error. However, the small errors in the wet gas reservoir model resulted in greater errors in the liquid and gas production forecasts. For example, based on the data set with $20 \%$ systematic and $0 \%$ random error, $4.92 \%$ and $4.04 \%$ error occurred for an estimated porosity and permeability, respectively, while the error rose to $14.13 \%$ for both liquid and gas production forecasts. As a conclusion, although systematic flow measurement errors might not have a significant effect on a wet gas reservoir model, they can affect its production forecasts considerably.

In the results of the wet gas reservoir, no meaningful trend is seen for the change in the parameter errors as a function of random errors. Although for porosity, similar to the results of the oil reservoir, when the random error has increased the effects of the systematic error have dampened, for the other three estimated parameters there is no clear trend in the change of the parameter errors when random error has changed. The effect of systematic errors in some cases has boosted and in other cases has been mitigated by increasing random errors. This observation is not surprising due to the nature of random errors. However, since the effect of the random errors is not considerable compared to the effect of systematic errors, in many cases it can be ignored without any significant change in the estimated results.

The significant effect of systematic errors on the final parameter estimations and production forecasts for both reservoirs shows the importance of the careful calibration and maintenance of flowmeters. As previously stated in the introduction, systematic errors can be prevented if the source of the error is found and eliminated. In contrast to systematic errors, although random errors can be reduced by installing new more precise flowmeters, this can be a costly exercise for oil and gas companies. The results of this study suggest that in terms of history matching and reservoir management, replacing current flowmeters with new ones might not be the best decision to improve the quality of observed production data. An alternative would be to invest in the regular calibration and maintenance of existing flowmeters, which would be a more effective and at the same time more economic decision. In addition to suggesting that regular calibration is valuable, the paper provides justifications (e.g. the possible cost of errors in history matching) to help in establishing 
a cash value for that recalibration in future, hence allowing better management decisions. This cash value may vary substantially for different fields and wells and may also lead to justifications for a different approach such as placing $1 \mathrm{~m}$ per well, or replacing one type of meter with another, or placing meters on specific high uncertainty wells. It can be an interesting topic for future research studies.

Recalibration and maintenance of flowmeters are already undertaken properly by many oil and gas companies based on their production protocols. However, the error in the production data that can affect history matching is not just caused by the flowmeters. In many oil and gas fields, production streams of different wells are commingled and only the total flow rate of all wells is measured by flowmeters. In these cases, the flow rates of individual wells are estimated by allocation calculations based on the results of occasional flow tests and the total flow rate of all wells. Allocation errors are normally larger than flowmeter errors and they can have a more significant effect on history matching. Increasing the regularity of flow tests or installing multiphase flowmeters on individual wells can reduce the systematic and random errors in the production data of individual wells and therefore reduce the uncertainty in the history matching process.

\section{Conclusions}

The results of the study clearly show the considerable effect of systematic flow measurement errors on the results of history matching. However, for the simulated oil and wet gas reservoir cases used in this study, the effect of random flow measurement errors on history matching was seen to be insignificant. Although systematic errors can be reduced by more careful calibration and maintenance of flowmeters, random errors are normally reduced by replacing an old flowmeter with a more precise one that as a consequence entails considerable expense for oil and gas producing companies. However, this study shows that particularly for history matching exercises, reducing random error does not lead to a consequent considerable reduction in the errors in the final results. Therefore, for the case of history matching, this study emphasises the importance of regular calibration and maintenance schedules for existing flowmeters as being a potentially more effective alternative to investing in replacing the flowmeters with new, more precise ones. Moreover, as the need for calibration is primarily to reduce systematic errors, it is important that the calibration is focussed on the actual operating range of the meter in its installed location.

Based on the results, history matching has been seen to be more sensitive to the flow measurement errors for an oil reservoir than for a wet gas reservoir. However, although the effect of flow measurement errors on the wet gas model parameters (i.e. porosity and permeability) has not been substantial, they have considerably affected the output parameters of the model (i.e. gas and liquid production forecast). In addition, there is normally a significant uncertainty in the production data of wet gas reservoirs due to the difficulty of measuring low fractions of liquid. This study shows that the effect of the uncertainty on the results of history matching for wet gas reservoirs can be noticeable.

Acknowledgements We would like to thank Fluid and Complex Systems Research Centre at Coventry University for the financial support for this work. We also appreciate the comments of Dr. Philip Costen in this article.

Open Access This article is distributed under the terms of the Creative Commons Attribution 4.0 International License (http://creativeco mmons.org/licenses/by/4.0/), which permits unrestricted use, distribution, and reproduction in any medium, provided you give appropriate credit to the original author(s) and the source, provide a link to the Creative Commons license, and indicate if changes were made.

\section{References}

Abdollahian A, Tadayoni M, Junin RB (2018) A new approach to reduce uncertainty in reservoir characterization using saturation height modeling, Mesaverde tight gas sandstones, western US basins. J Pet Explor Prod Technol. https://doi.org/10.1007/s1320 2-018-0594-5

Ahmed TH (1989) Hydrocarbon phase behavior, 7. Gulf Pub Co

Al-Hussainy R, Humphreys N (1996) Reservoir management: principles and practices. J Petrol Technol 48(12):1129-1135

Babak O, Deutsch C (2008) Reserves uncertainty calculation accounting for parameter uncertainty. J Canad Pet Technol 47(08):41-49

Barros E, Van den Hof P, Jansen JD (2016) Value of information in closed-loop reservoir management. Comput Geosci 20(3):737-749

Bergren FE, Lagerlef DL, Feldman S (1997) Three-phase welllevel production allocation at Prudhoe Bay. SPE Comput Appl 9(02):55-60

Chakra NC, Saraf DN (2016) History matching of petroleum reservoirs employing adaptive genetic algorithm. J Pet Explor Prod Technol 6(4):653-674

Cramer R, Schotanus D, Ibrahim K, Colbeck N (2011) Improving allocation and hydrocarbon accounting accuracy using new techniques. SPE Econ Manag 3(04):235-240

Dandekar AY (2013) Petroleum reservoir rock and fluid properties. CRC Press, Boca Raton

Energy Institute (2012) HM 96: Guidelines for the allocation of fluid streams in oil and gas production, London

Falcone G, Hewitt G, Alimonti C, Harrison B (2002) Multiphase flow metering: current trends and future developments. J Petrol Technol 54(04):77-84

Falcone G, Hewitt G, Alimonti C (2009) Multiphase flow metering: principles and applications, vol 54. Elsevier, Amsterdam

Guidance Notes for Petroleum Measurement (2015) Oil and gas authority, UK, p 77

Habib M, Guangqing Y, Xie C, Charles SP, Jakada H, Danlami MS, Ahmed HA, Omeiza IA (2017) Optimizing oil and gas field management through a fractal reservoir study model. J Pet Explor Prod Technol 7(1):43-53

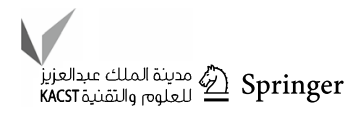


Hamdi H, Behmanesh H, Clarkson CR, Sousa MC (2015) Using differential evolution for compositional history-matching of a tight gas condensate well in the Montney Formation in western Canada. J Nat Gas Sci Eng 26:1317-1331

Hanssen KG, Codas A, Foss B (2017) Closed-loop predictions in reservoir management under uncertainty. SPE J 22:1-585

Jansen J-D, Brouwer D, Naevdal G, Van Kruijsdijk C (2005) Closedloop reservoir management. First Break 23(1):43-48

Jansen J-D, Brouwer R, Douma SG (2009) Closed loop reservoir management. In: SPE Reservoir simulation symposium. Society of Petroleum Engineers

Kaiser MJ (2014) Multiple well lease decomposition and forecasting strategies. J Petrol Sci Eng 116:59-71

Kirsch A (2011) An introduction to the mathematical theory of inverse problems, vol 120. Springer, Berlin

Leeson TJ, Heering J, Dykesteen E (2001) Temporary deployment of multiphase flow measurement to provide well performance data: a case study, offshore technology conference. In: Offshore technology conference

Letton C, Hall AR (2012) Multiphase and wet gas flow measurementit's not that simple. In: Abu Dhabi international petroleum conference and exhibition. Society of Petroleum Engineers

Lindsay G, Hay J, Glen N, Shariatipour S (2017) Profiling and trending of coriolis meter secondary process value drift due to ambient temperature fluctuations. Flow Meas Instrum 59:225-232

Liu F, Darjani S, Akhmetkhanova N, Maldarelli C, Banerjee S, Pauchard V (2017) Mixture effect on the dilatation rheology of asphaltenes-laden interfaces. Langmuir 33(8):1927-1942

Lorentzen RJ, Shafieirad A, Naevdal G (2009) Closed loop reservoir management using the ensemble Kalman filter and sequential quadratic programming. In: SPE Reservoir simulation symposium. Society of Petroleum Engineers

Makhlouf EM, Chen WH, Wasserman ML, Seinfeld JH (1993) A general history matching algorithm for three-phase, three-dimensional petroleum reservoirs. SPE Adv Technol Ser 1(02):83-92

Marshall CD, Sadri M, Hamdi H, Shariatipour SM, Lee WK, Thomas A, Shaw-Stewart J (2018) The role of flow measurement in hydrocarbon recovery forecasting in the UKCS. J Porous Media (in press)

McCain WD (1990) The properties of petroleum fluids. PennWell Books, Tulsa

Mozaffari S, Tchoukov P, Mozaffari A, Atias J, Czarnecki J, Nazemifard N (2017) Capillary driven flow in nanochannels-application to heavy oil rheology studies. Colloids Surf A Physicochem Eng Asp 513:178-187
Obidegwu D, Chassagne R, MacBeth C (2017) Seismic assisted history matching using binary maps. J Nat Gas Sci Eng 42:69-84

Oliver DS, Chen Y (2011) Recent progress on reservoir history matching: a review. Comput Geosci 15(1):185-221

Oliver DS, Reynolds AC, Liu N (2008) Inverse theory for petroleum reservoir characterization and history matching. Cambridge University Press, Cambridge

Pobitzer A, Skålvik AM, Bjørk RN (2016) Allocation system setup optimization in a cost-benefit perspective. J Petrol Sci Eng 147:707-717

Sadri M, Shariatipour S, Hunt A (2017) Effects of flow measurement errors on oil and gas production forecasts. Comput Exp Methods Multiph Complex Flow IX 115:133

Satter A, Varnon JE, Hoang MT (1994) Integrated reservoir management. J Petrol Technol 46(12):1057-1064

Stephen KD, Macbeth C (2008) Reducing reservoir prediction uncertainty by updating a stochastic model using seismic history matching. SPE Reservoir Eval Eng 11(06):991-999

Tavassoli Z, Carter JN, King PR (2004) Errors in history matching. SPE J 9(03):352-361

Taylor J (1997) Introduction to error analysis, the study of uncertainties in physical measurements. University Science Books, New York

Thakur GC (1996) What is reservoir management? J Petrol Technol 48(06):520-525

Tombs M, Henry M, Zhou F, Lansangan RM, Reese M (2006) High precision Coriolis mass flow measurement applied to small volume proving. Flow Meas Instrum 17(6):371-382

Trice M Jr, Dawe B (1992) Reservoir management practices. J Petrol Technol 44(12):1344-1349

Tunnish A, Shirif E, Henni A (2018) History matching of experimental and CMG-STARS results. J Pet Explor Prod Technol 9(1):341-351

Wang C, Li G, Reynolds AC (2009) Production optimization in closedloop reservoir management. SPE J 14(03):506-523

Xu B, Wu Y, Cheng L, Huang S, Bai Y, Chen L, Liu Y, Yang Y, Yang L (2018) Uncertainty quantification in production forecast for shale gas well using a semi-analytical model. J Pet Explor Prod Technol. https://doi.org/10.1007/s13202-018-0598-1

Publisher's Note Springer Nature remains neutral with regard to jurisdictional claims in published maps and institutional affiliations. 